\section{R. Adkins* \\ C. E. Brennen}

California Institute of Technology, Pasadena, CA 91125

\title{
Analyses of Hydrodynamic Radial Forces on Centrifugal Pump Impellers
}

Hydrodynamic interactions that occur between a centrifugal pump impeller and a volute are experimentally and theoretically investigated. The theoretical analysis considers the inability of the blades to perfectly guide the flow through the impeller, and also includes a quasi-one dimensional treatment of flow in the volute. Flow disturbances at the impeller discharge and the resulting forces are determined by the theoretical model. The model is then extended to obtain the hydrodynamic force perturbations that are caused by the impeller whirling eccentrically in the volute. Under many operating conditions, these force perturbations were found to be destabilizing. Comparisons are made between the theoretical model and the experimental measurements of pressure distributions and radial forces on the impeller. The theoretical model yields fairly accurate predictions of the radial forces caused by the flow through the impeller. However, it was found that the pressure acting on the front shroud of the impeller has a substantial effect on the destabilizing hydrodynamic forces.

\section{Introduction}

This study investigates the forces that result from the hydrodynamic interaction between the impeller and the volute in a centrifugal pump. Figure 1 shows a common type of centrifugal pump with a few of the key components identified. Volutes are usually constructed to provide a uniform impeller discharge when the pump operates at design conditions. However, the discharge flow pattern will no longer be uniform at off-design conditions. Once the flowrate changes, the discharge conditions around the impeller become asymmetric for any given volute. Even at the volute design flowrate, the discharge conditions could still become asymmetric if the impeller is displaced from the "design" center of the volute by shaft deflection, bearing wear, etc. In either case, a net radial force on the impeller will result from the asymmetric discharge conditions.

It is customary in rotordynamic analyses to linearize the radial forces acting on the rotor in terms of a steady portion acting on the centered impeller, and a time dependent part caused by the impeller whirling. Here it will be assumed that the impeller whirls in a small circular orbit. Referring to Fig. 1 , these terms may be expressed as,

$$
\left\{\begin{array}{l}
F_{x}^{*} \\
F_{y}^{*}
\end{array}\right\}=\left\{\begin{array}{l}
\bar{F}_{x} \\
\bar{F}_{y}
\end{array}\right\}+\left[\begin{array}{ll}
A_{x x}^{*} & A_{x y}^{*} \\
A_{y x}^{*} & A_{y y}^{*}
\end{array}\right]\left\{\begin{array}{l}
\epsilon^{*} \cos \omega t \\
\epsilon^{*} \sin \omega t
\end{array}\right\},
$$

where $\bar{F}_{x}$ and $\bar{F}_{y}$ result from the interaction of the centered impeller with the volute, and the matrix $[A]$ relates the perturbed

\footnotetext{
*Now at Sandia National Laboratories, Albuquerque, NM 87185.

Contributed by the Fluids Engineering Division for publication in the JourNal of Fluins Engineering. Manuscript received by the Fluids Engineering
} Division September 29, 1986. force to the eccentric position of the impeller. The $[A]$ matrix will be a function of the whirl speed, $\omega$, and is often expressed as a quadratic in $\omega$ so that the system resembles a simple stiffness, damping, and mass model.

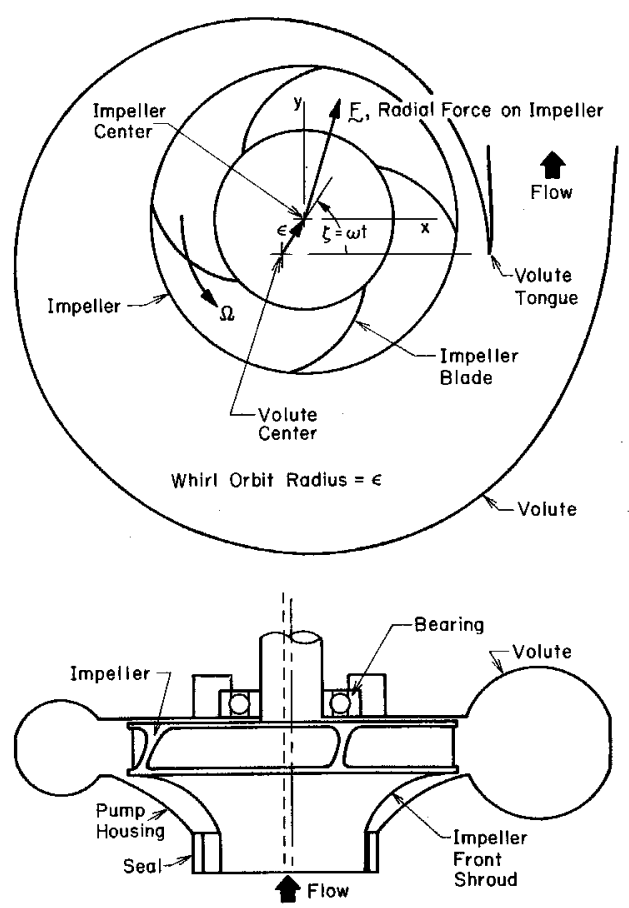

Fig. 1 Description of a centrifugal pump 
The steady or mean forces, $\bar{F}_{x}$ and $\bar{F}_{y}$, have been examined in several studies and an understanding of them has been greatly enhanced through papers by Iversen et al. (1960), Csanady (1962), and Agostinelli et al. (1960) to name a few. All of these authors have shown that there is a particular flowrate where forces on the impeller will be minimized for a given volute. Previous experimental (Chamieh et al., 1982, and Jery et al., 1984) and theoretical (Colding-Jorgensen, 1980) investigations have also shown that the components of $[A]$ are such that a whirling motion of the impeller would be encouraged rather than dissipated by the hydrodynamic effects. This finding has generated concern that the rotor assembly may whirl at one of its natural frequencies even though the shaft may be rotating well above this speed. If the impeller whirls at a subsynchronous speed, the shaft will be subjected to alternating flexural stresses that can cause metal fatigue (see Ehrich and Childs, 1984).

In the current study, a theoretical model of the volute and impeller flows will be developed and compared to experimental results. Previously, a potential flow model for the steady forces on a centered impeller was given by Csanady (1962) and this work was later extended by Colding-Jorgensen (1980) to include the effects of the impeller whirling within the volute. Two dimensional potential flow models for whirling impellers have also been developed by Shoji and Ohashi [1980] and [1987]. In potential flow models, however, problems arise in relating the two dimensional theoretical volute profiles to the three dimensional geometries of real volutes. To avoid this problem, the model developed in this paper uses a bulk flow description of the volute flow which can use measured volute geometries directly. A similar treatment of the volute flow was presented by Iversen et al. (1960), but the influence of this flow on the impeller discharge conditions was largely ignored and only the non-whirling impeller was considered. The impeller/volute interaction will be included along with the effects of impeller whirl in the present analysis.

\section{Theoretical Analysis}

In developing the current theoretical model, the problem is broken into its two natural parts; models are constructed for the flow through the impeller and in the volute. The equations that are generated in these two parts are then combined by matching the pressures and velocities at the impeller discharge to those at the volute inlet. A full development of this model is given by Adkins (1985) and only a brief summary will be presented here.

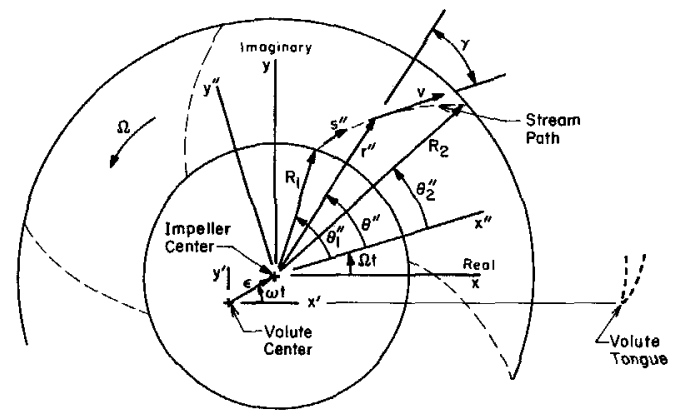

Fig. 2 Geometry of a centrifugal pump impeller

Governing Equations for the Impeller. Figure 2 illustrates the geometries used in developing the impeller model. To relate the pressure between the inlet and discharge of the impeller, a simplified unsteady form of Bernoulli's equation is written as,

$$
\begin{aligned}
& \frac{P_{i}}{\rho}+\frac{v^{2}}{2}-\frac{\Omega^{2} r^{\prime \prime 2}}{2} \\
& \quad+\int_{s} \frac{\partial v}{\partial t} d s^{\prime \prime}-\omega^{2} \epsilon \int_{s^{\prime \prime}} \cos \left(\omega t-\Omega t-\theta^{\prime \prime}\right) d r^{\prime \prime} \\
& \quad-\omega^{2} \epsilon \int_{s^{\prime \prime}} \sin \left(\omega t-\Omega t-\theta^{\prime \prime}\right) r^{\prime \prime} d \theta^{\prime \prime}=F(t)
\end{aligned}
$$

Here the flow is assumed to be two dimensional and the impeller whirl speed constant.

To simplify the model, certain assumptions must be made about the velocity field within the impeller. Specifically, the flow in the impeller is assumed to follow a spiral path with inclination angle, $\gamma$, which is fixed relative to the impeller for a given flowrate and head rise so that,

$$
\theta_{2}^{\prime \prime}=\theta^{\prime \prime}+\tan \gamma \ln \left(r^{\prime \prime} / R_{2}\right)
$$

Here $\left(r^{\prime \prime}, \theta^{\prime \prime}\right)$ and $\left(R_{2}, \theta_{2}^{\prime \prime}\right)$ are the coordinates of a general point on a streamline within the impeller and at the position of discharge respectively. The flow path angle, $\gamma$, of the streamlines is permitted to deviate from the impeller blade angle and it is found by equating theoretical and experimental head/flowrate characteristics (see Closure Conditions). To account for the impeller flow asymmetry caused by the volute, a circumferential perturbation is superimposed on the mean impeller flow. This flow perturbation is assumed to be stationary $b=$ width of impeller discharge passage

$h=$ total head $\left(h^{*}=2 h / \rho \Omega^{2} R_{2}^{2}\right)$

$j=\sqrt{-1}$

$\mathbf{k}=$ impeller phase coefficient $=\cos (\tan \gamma \ln$ $(R))+j \sin (\tan \gamma \ln (R))$

$r, \theta=$ polar coordinate system

$s=$ length in tangential direction

$t=$ time

$v=$ relative velocity in impeller

$w=$ width in volute

$\bar{A}, \overline{r A}, \overline{r r A}$

$x, y, z=$ rectangular coordinate system

$$
\begin{aligned}
\overline{\ln r A}, \overline{\ln r A}= & \begin{array}{l}
\text { moments of volute cross-sectional area } \\
\text { (defined in equations }(14 a-e))
\end{array} \\
A_{i j}= & (i=x, y, j=x, y) \text { components of } \\
& \text { generalized hydrodynamic force matrix }[A] \\
& \left(A_{i j}^{*}=A_{i j} / \rho \pi b \Omega^{2} R_{2}^{3}\right) \\
C_{i j}= & (i=x, y, j=x, y) \text { components of damping } \\
& \text { force matrix }[C]\left(C_{i j}^{*}=C_{i j} / \rho \pi b \Omega R_{2}^{2}\right)
\end{aligned}
$$

$$
\begin{aligned}
D_{p}= & \text { pressure coefficient at volute inlet }= \\
& 2\left(P_{v}\left(R_{2}^{\prime}, \theta^{\prime}\right)-h_{1}\right) / \rho \Omega^{2} R_{2}^{2} \\
F= & \text { force acting on impeller }\left(F^{*}=F / \rho \pi b \Omega^{2} R_{2}^{3}\right) \\
F(t)= & \text { integration constant in Bernoulli's equation } \\
J_{i j}= & (i=x, y, j=x, y) \text { coefficients of the jerk } \\
& \text { force matrix }[J]\left(J_{i j}^{*}=J_{i j} / \rho \pi b R_{2}^{2} / \Omega\right) \\
K_{i j}= & (i=x, y, j=x, y) \text { components of stiffness } \\
& \text { force matrix }[K]\left(K_{i j}^{*}=K_{i j} / \rho \pi b \Omega^{2} R_{2}^{2}\right) \\
M_{i j}= & (i=x, y, j=x, y) \text { components of inertia } \\
& \text { force matrix }[M]\left(M_{i j}^{*}=M_{i j} / \rho \pi b R_{2}^{2}\right) \\
P_{i}= & \text { pressure in impeller }\left(P_{i}^{*}=2 P P_{i} / \rho \Omega^{2} R_{2}^{2}\right) \\
P_{v}= & \text { pressure in volute }\left(P=2 P_{v}^{*} / \rho \Omega^{2} R_{2}^{2}\right) \\
R= & \text { impeller radius (with no subscript, } R= \\
& \left.R_{2} / R_{1}\right) \\
R_{t}= & \text { radius of pressure tap ring } \\
V= & \text { velocity in volute (with no subscript, } V^{*}= \\
& \left.V_{\theta} / \Omega R_{2}\right) \\
W_{i}= & \text { external width of impeller at } R_{2}\left(W_{i}^{*}=\right. \\
& \left.W_{i} / b\right) \\
\beta= & \text { perturbation function for impeller flow }
\end{aligned}
$$


in the volute reference frame. Together, these stipulations require that,

$$
v=\left(v_{r}^{2}+v_{\theta}^{2}\right)^{1 / 2}=\phi \Omega R_{2}^{2} \beta\left(\theta^{\prime \prime}, r^{\prime \prime}, \Omega t, \omega t, \epsilon\right) \sec \gamma / r^{\prime \prime} .
$$

From continuity considerations, the perturbation function, $\beta$, must be constant along a streamline. For whirl motions with small eccentric orbits, $\beta$ may be linearized as,

$\beta\left(\theta^{\prime \prime}, r^{\prime \prime}, \Omega t, \omega t, \epsilon\right)=\bar{\beta}\left(\theta_{2}\right)+\epsilon^{*}\left\{\beta_{c}\left(\theta_{2}\right) \cos \omega t+\beta_{s}\left(\theta_{2}\right) \sin \omega t\right\}$.

Equations (4) and (5) can now be substituted into equation (2). The pressure at the impeller discharge is then given as a function of $\beta$ and the inlet pressure. If it assumed that the total head is circumferentially constant at the impeller inlet and there is no inlet pre-swirl, the inlet pressure can be written as,

$$
\begin{aligned}
& P_{i}^{*}\left(R_{1}, \theta_{1}\right) \cong h_{1}^{*}-\phi R \bar{\beta}\left(\theta_{2}\right)\left\{\phi R \bar{\beta}\left(\theta_{2}\right)+2 \epsilon^{*} \frac{\omega}{\Omega} \sin \left(\theta_{1}-\omega t\right)\right\} \\
&-2 \epsilon^{*} \phi^{2} R^{2} \bar{\beta}\left(\theta_{2}\right)\left\{\beta_{c}\left(\theta_{2}\right) \cos \omega t+\beta_{s}\left(\theta_{2}\right) \sin \omega t\right\},
\end{aligned}
$$

for small eccentric displacements. By utilizing equations (4) through (6), and neglecting terms of order $\epsilon^{2}$ and higher, Bernoulli's equation can now be separated into harmonics with steady, $\epsilon \cos \omega t$, and $\epsilon \sin \omega t$ dependence as,

$$
\phi \sec ^{2} \gamma\left[2 \ln (R) \frac{d \bar{\beta}}{d \theta_{2}}+\phi \bar{\beta}^{2}\right]+\bar{D}_{p}-1=0,
$$

$$
\begin{gathered}
2 \phi \sec ^{2} \gamma\left[\ln (R) \frac{d \beta_{c}}{d \theta_{2}}+\phi \bar{\beta} \beta_{c}+\frac{\omega}{\Omega} \ln (R) \beta_{s}\right] \\
+D_{p c}-\sin \theta_{2} \frac{d \bar{D}_{p}}{d \theta_{2}}+2 \frac{\omega}{\Omega}\left[\phi R \bar{\beta} \sin \left(\theta_{2}+\tan \gamma \ln (R)\right)\right. \\
\left.-\cos \left(\theta_{2}+\tan \gamma \ln (R)\right) / R\right]-2 \frac{\omega^{2}}{\Omega^{2}}\left[\cos \theta_{2}\right. \\
\left.-\cos \left(\theta_{2}+\tan \gamma \ln (R)\right) / R\right] / \tan ^{2} \gamma=0 \\
2 \phi \sec ^{2} \gamma\left[\ln (R) \frac{d \beta_{s}}{d \theta_{2}}+\phi \bar{\beta} \beta_{s}-\frac{\omega}{\Omega} \ln (R) \beta_{c}\right] \\
+D_{p s}+\cos \theta_{2} \frac{d \bar{D}_{p}}{d \theta_{2}}-2 \frac{\omega}{\Omega}\left[\phi R \bar{\beta} \cos \left(\theta_{2}+\tan \gamma \ln (R)\right)\right. \\
\left.+\sin \left(\theta_{2}+\tan \gamma \ln (R)\right) / R\right]-2 \frac{\omega^{2}}{\Omega^{2}}\left[\sin \theta_{2}\right. \\
\left.-\sin \left(\theta_{2}+\tan \gamma \ln (R)\right) / R\right] / \tan ^{2} \gamma=0
\end{gathered}
$$



Fig. 3 Geometry of a volute

where,

$$
D_{p}\left(\theta^{\prime}\right)=\overline{D_{p}}\left(\theta^{\prime}\right)+\epsilon^{*}\left[D_{p c}\left(\theta^{\prime}\right) \cos \omega t+D_{p s}\left(\theta^{\prime}\right) \sin \omega t\right] .
$$

In equations $(7 a-c)$ the volute inlet pressure coefficient, $D_{p}\left(\theta^{\prime}\right)$, has been transformed into the impeller reference frame by the approximation, $\theta_{2}=\theta^{\prime}+\epsilon^{*} \sin \left(\theta^{\prime}-\omega t\right)$. This will prove convenient in the future, because the impeller discharge pressure will be equated with the volute inlet pressure.

Governing Equations for the Volute. The geometries used in developing the volute model are shown in Fig. 3. The volute flow will be described by a continuity equation, a moment of momentum equation, and an equation of motion in the radial direction. Each of these three equations can be written respectively as,

$$
\begin{array}{r}
\frac{\partial\left(w V_{\theta^{\prime}}\right)}{\partial \theta^{\prime}}+\frac{\partial\left(w r^{\prime} V_{r^{\prime}}\right)}{\partial r^{\prime}}=0, \\
\frac{\partial\left(w r^{\prime} V_{\theta^{\prime}} V_{\theta^{\prime}}\right)}{\partial \theta^{\prime}}+\frac{\partial\left(w r^{\prime} r^{\prime} V_{\theta^{\prime}} V_{r^{\prime}}\right)}{\partial r^{\prime}} \\
+w r^{\prime} r^{\prime} \frac{\partial V_{\theta^{\prime}}}{\partial t}=-\frac{w r^{\prime}}{\rho} \frac{\partial P_{v}}{\partial \theta^{\prime}},
\end{array}
$$

and,

$$
\frac{\partial P_{v}}{\partial r^{\prime}}=\frac{\rho V_{\theta^{\prime}} V_{\theta^{\prime}}}{r^{\prime}}
$$

Here it has been estimated that $V_{r^{\prime}}$ and $V_{z^{\prime}}$ (and their gradients) are much smaller than $V_{\theta^{\prime}}$, except at the inlet of the volute.

\section{Nomenclature (cont.)}

$$
\begin{aligned}
\zeta= & \text { angular location of the impeller center } \\
& (=\omega t=\text { constant }) \\
\gamma= & \text { angle of flow path through impeller } \\
\epsilon= & \text { distance between impeller and volute } \\
& \text { centers }\left(\epsilon^{*}=\epsilon / R_{2}\right) \\
\rho= & \text { fluid density } \\
\phi= & \text { flow coefficient }=\text { flowrate through } \\
& \text { pump } / 2 \pi b \Omega R_{2}^{2} \\
\psi= & \text { total head rise coefficient }=\left(h_{d}-\right. \\
& \left.h_{1}\right) / \rho \Omega^{2} R_{2}^{2} \\
\omega= & \text { orbit speed of impeller center (whirl speed) } \\
\Omega= & \text { rotational speed of impeller (shaft speed) }
\end{aligned}
$$

\section{Subscripts}

$$
\begin{aligned}
c, s= & \cos \omega t \text { and sin } \omega t \text { components } \\
& \text { (nondimensional) } \\
d= & \text { downstream of pump } \\
\exp = & \text { experimental result } \\
m= & \text { force component due to momentum } \\
& \text { exchange }
\end{aligned}
$$

$$
\begin{aligned}
p= & \text { force component due to pressure } \\
r, \theta= & \text { radial or angular component } \\
x, y= & \text { components in rectangular directions (real } \\
& =x \text { and imaginary }=y \text { ) } \\
1,2= & \text { impeller inlet and discharge }
\end{aligned}
$$

Superscripts

$$
\begin{aligned}
& \text { = measurement made in volute reference } \\
& \text { frame } \\
& "=\text { measurement made from frame fixed to } \\
& \text { rotating impeller } \\
& \text { * }=\text { nondimensionalized quantity }
\end{aligned}
$$

\section{Special Notation}

$$
\begin{aligned}
\mathbf{F}= & \text { bold type denotes vector quantity } \\
\bar{V}= & \text { overbar denotes centered impeller value } \\
& \text { (nondimensional) } \\
{[A]=} & \text { square brackets denote a matrix quantity } \\
\dot{x}= & \text { dot represents a time derivative }
\end{aligned}
$$


Flow within the volute is considered to be primarily in the $\theta^{\prime}$ direction and the velocity profile is assumed to be flat. This treatment will allow equations (9), (10), and (11) to be integrated over the volute cross-section. When these equations are combined with equations (4) and (5), the pressure and velocity distributions in the volute will be given in terms of moments of the volute cross-sectional area and the perturbation function, $\beta$. Both equations (9) and (10) can then be separated into three parts (steady, $\epsilon \cos \omega t$, and $\epsilon \sin \omega t$ ) as follows:

Continuity:

$$
\frac{d(\bar{V} \bar{A})}{d \theta^{\prime}}=\phi \bar{\beta}
$$

$\frac{d\left(V_{c} \bar{A}\right)}{d \theta^{\prime}}=W_{i}^{*} \frac{d\left(\bar{V} \cos \theta^{\prime}\right)}{d \theta^{\prime}}$

$$
+\frac{\omega}{\Omega} \sin \theta^{\prime}+\phi\left[\beta_{c}+\frac{d\left(\bar{\beta} \sin \theta^{\prime}\right)}{d \theta^{\prime}}\right],
$$

$\frac{d\left(V_{s} \bar{A}\right)}{d \theta^{\prime}}=W_{i}^{*} \frac{d\left(\bar{V} \sin \theta^{\prime}\right)}{d \theta^{\prime}}$

$$
-\frac{\omega}{\Omega} \cos \theta^{\prime}+\phi\left[\beta_{s}-\frac{d\left(\bar{\beta} \cos \theta^{\prime}\right)}{d \theta^{\prime}}\right] \text {, }
$$

Moment of momentum:

$$
\begin{aligned}
\frac{\overline{r A}}{2} \frac{d \overline{D_{p}}}{d \theta^{\prime}} & =-\frac{d\left(\overline{r A} \bar{V}^{2}\right)}{d \theta^{\prime}} \\
& -\overline{r \ln r A} \frac{d\left(\bar{V}^{2}\right)}{d \theta^{\prime}}+\phi(1-\phi \tan \gamma \bar{\beta}) \bar{\beta},
\end{aligned}
$$

$$
\frac{\overline{r A}}{2} \frac{d D_{p c}}{d \theta^{\prime}}=-2 \frac{d\left(\overline{r A} \bar{V} V_{c}\right)}{d \theta^{\prime}}
$$

$$
\begin{gathered}
+\frac{\omega}{\Omega}\left(W_{i}^{*} \sin \theta^{\prime} \bar{V}-\overline{r r A} V_{s}\right) \\
+\left(W_{i}^{*}+\overline{r A}\right) \frac{d\left(\bar{V}^{2} \cos \theta^{\prime}\right)}{d \theta^{\prime}}-2 \overline{r \ln r A} \frac{d\left(\bar{V} V_{c}\right)}{d \theta^{\prime}} \\
+\phi^{2} \sin \theta^{\prime} \bar{\beta}^{2}+\phi \cos \theta^{\prime}\left(\frac{\omega}{\Omega}+2-2 \phi \tan \gamma \bar{\beta}\right) \bar{\beta}
\end{gathered}
$$

$+\phi(1-2 \phi \tan \gamma \bar{\beta})\left(\beta_{c}+\sin \theta^{\prime} \frac{d \bar{\beta}}{d \theta^{\prime}}\right)+\frac{W_{i}^{*}}{2} \cos \theta^{\prime} \frac{d \overline{D_{p}}}{d \theta^{\prime}}$,

$$
\begin{aligned}
& \frac{\overline{r A}}{2} \frac{d D_{p s}}{d \theta^{\prime}}=-2 \frac{d\left(\overline{r A} \bar{V} V_{s}\right)}{d \theta^{\prime}} \\
& \quad-\frac{\omega}{\Omega}\left(W_{i}^{*} \cos \theta^{\prime} \bar{V}-\overline{r r} \bar{A} V_{c}\right) \\
& +\left(W_{i}^{*}+\overline{r A}\right) \frac{d\left(\bar{V}^{2} \sin \theta^{\prime}\right)}{d \theta^{\prime}}-2 \overline{r \ln r A} \frac{d\left(\bar{V} V_{s}\right)}{d \theta^{\prime}} \\
& \quad-\phi^{2} \cos \theta^{\prime} \bar{\beta}^{2}+\phi \sin \theta^{\prime}\left(\frac{\omega}{\Omega}+2-2 \phi \tan \gamma \bar{\beta}\right) \bar{\beta} \\
& +\phi(1-2 \phi \tan \gamma \bar{\beta})\left(\beta_{s}-\cos \theta^{\prime} \frac{d \bar{\beta}}{d \theta^{\prime}}\right)+\frac{W_{i}^{*}}{2} \sin \theta^{\prime} \frac{d \overline{D_{p}}}{d \theta^{\prime}},
\end{aligned}
$$

where,

$$
\begin{aligned}
& \bar{A}\left(\theta^{\prime}\right)=\int_{R_{2}}^{R_{3}^{\prime}} w d r^{\prime} / b R_{2}, \\
& \overline{\ln r A}\left(\theta^{\prime}\right)=\int_{R_{2}}^{R_{3}^{\prime}} \ln \left(r^{\prime} / R_{2}\right) w d r^{\prime} / b R_{2}, \\
& \overline{r A}\left(\theta^{\prime}\right)=\int_{R_{2}}^{R_{3}^{\prime}} r^{\prime} w d r^{\prime} / b R_{2}^{2}, \\
& \overline{r r A}\left(\theta^{\prime}\right)=\int_{R_{2}}^{R_{3}^{\prime}} r^{\prime} r^{\prime} w d r^{\prime} / b R_{2}^{3}, \\
& \overline{r \ln r A}\left(\theta^{\prime}\right)=\int_{R_{2}}^{R_{3}^{\prime}} r^{\prime} \ln \left(r^{\prime} / R_{2}\right) w d r^{\prime} / b R_{2}^{2},
\end{aligned}
$$

$(14 a-e)$

and,

$V^{*}\left(\theta^{\prime}\right)=\bar{V}\left(\theta^{\prime}\right)+\epsilon^{*}\left[V_{c}\left(\theta^{\prime}\right) \cos \omega t+V_{s}\left(\theta^{\prime}\right) \sin \omega t\right] \cong \frac{V_{\theta}^{\prime}}{\Omega R_{2}}$.

In equations $(12 a-c)$ and $(13 a-c)$ the perturbation function, $\beta$, has been transformed into the volute reference frame for convenience in obtaining a solution.

To complete the basic equations for the volute problem, equation (11) may be integrated to give the radial pressure variation in the volute as,

$$
\begin{aligned}
P_{v}^{*}=\overline{D_{p}}+2 \bar{V}^{2} & {\left[\ln \left(r^{\prime} / R_{2}\right)-\epsilon^{*} \cos \left(\theta^{\prime}-\omega t\right)\right] } \\
& +\epsilon^{*}\left[D_{p c}+4 \bar{V} V_{c} \ln \left(r^{\prime} / R_{2}\right)\right] \cos \omega t \\
& +\epsilon^{*}\left[D_{p s}+4 \bar{V} V_{s} \ln \left(r^{\prime} / R_{2}\right)\right] \sin \omega t
\end{aligned}
$$

Closure Conditions. Equations (7), (12), (13), and (16) will describe the flow in the impeller and the volute after certain boundary conditions are satisfied. Even though $\beta$ is referred to as the perturbation function, it was never assumed to be small. However, from the definition of the flow coefficient, $\beta$ is required to have an average of one. The flow perturbation is further assumed to possess at least zeroth order continuity around the periphery of the impeller. This restriction on $\beta$ can be met by satisfying the condition,

$$
\beta\left(R_{2}, 0\right)=\beta\left(R_{2}, 2 \pi\right),
$$

At the tongue of the volute, it is assumed that the average total head of the recirculated flow will be constant across the tongue, so that,

$$
\begin{aligned}
\left.\int_{R_{2}^{\prime}(0)}^{R_{3}^{\prime}(0)}\left(P_{v}+\rho V_{\theta^{\prime}}^{2} / 2\right)\right|_{\theta^{\prime}=2 \pi} w d r^{\prime} \\
=\left.\int_{R_{2}^{\prime}(0)}^{R_{3}^{\prime}(0)}\left(P_{v}+\rho V_{\theta^{\prime}}^{2} / 2\right)\right|_{\theta^{\prime}=0} w d r^{\prime},
\end{aligned}
$$

From the remaining flow that is discharged, the flow path angle, $\gamma$, will be determined. This angle will vary with flowrate and total head and it can be found by equating the predicted and experimental total head rise across the pump as,

$$
\psi_{\exp }=\psi=\left[\overline{D_{p}}(2 \pi)+C_{v} \bar{V}^{2}(2 \pi)\right] / 2,
$$

where,

$$
C_{v}=1+2[\overline{\ln r A}(2 \pi)-\overline{\ln r A}(0)] /[\bar{A}(2 \pi)-\bar{A}(0)] .
$$

Admittedly, using an experimental result does limit the preliminary design applications of this model. However, the " $H / Q$ " curve (in dimensionless form the function $\psi_{\exp }(\phi)$ ) is normally available for any pump and it is important that this fundamental characteristic is properly represented in the model.

This completes the development of equations necessary to determine the flow properties, $\beta, D_{p}, V$, and $\gamma$. However, 
some guidance on obtaining a solution is needed. Volute properties specified in equation (14a-e) are introduced as second order spline curve-fits of measured volute dimensions. The nine ordinary differential equations (7), (12), and (13) are written in a centered difference form and solved in an iterative manner. Steady flow properties are obtained as follows:

(a) Assume $\bar{\beta}\left(\theta_{2}\right)=1$ and choose $\bar{D}_{p}(2 \pi)-\bar{D}_{p}(0)$ and $\gamma$.

(b) Calculate $\bar{V}(0)$ using the steady part equation (16) combined with equation (18). Solve equation (12a) for $\bar{V}\left(\theta^{\prime}\right)$.

(c) Solve equation (13a) for $\bar{D}_{p}\left(\theta^{\prime}\right) . \bar{D}_{p}(0)$ is chosen so that the 0 to $2 \pi$ integral of equation $(7 a)$ is satisfied.

(d) If $\bar{D}_{p}(2 \pi)-\bar{D}_{p}(0)$ does not match the original estimate, update the estimate and return to step $(b)$.

(e) Solve for $\bar{\beta}\left(\theta_{2}\right)$ using equation (7a) and the closure condition in equation (17).

(f) If $\bar{\beta}\left(\theta_{2}\right)$ does not match the original estimate, update the estimate on $\bar{\beta}\left(\theta_{2}\right)$ and return to step (c). (Note, the average of $\bar{\beta}$ must equal one.)

(g) When $\bar{\beta}\left(\theta_{2}\right)$ has converged, check that equation (19) is satisfied. If this condition is not met, return to step $(b)$ with a new estimate of $\gamma$.

(h) After equation (19) is satisfied, the solution has converged and $\bar{\beta}, \bar{D}_{p}, \bar{V}$, and $\gamma$ are known.

The numerical solution was tested using exact solutions for sinusoidal $\bar{\beta}$ distributions. Flow properties for an off-centered impeller were obtained with a similar iterative procedure except $\gamma$ is assumed to be fixed by the steady flow computations. For a whirling impeller, the cos $\omega t$ and sin $\omega t$ terms become coupled and parts $(b)$ and $(c)$ of equations (7), (12), and (13) must be solved simultaneously.

Hydrodynamic Forces on the Impeller. Basically, there are two sources that contribute to the radial hydrodynamic forces on an impeller. One part is due to an asymmetric pressure distribution around the impeller and the other part is caused by the asymmetric momentum fluxes at the impeller inlet and discharge. The first contribution is evaluated by integrating the pressure around the inlet and discharge of the impeller:

$$
\begin{gathered}
\mathbf{F}_{p}=\left(F_{x}+j F_{y}\right)_{p}=b \int_{0}^{2 \pi} P_{i}\left(R_{1}, \theta_{1}\right) R_{1} e^{j \theta_{1}} d \theta_{1} \\
-W_{i} \int_{0}^{2 \pi} P_{i}\left(R_{2}, \theta_{2}\right) R_{2} e^{j \theta_{2}} d \theta_{2},
\end{gathered}
$$

where $j$ denotes the imaginary part that corresponds to the $y$ direction (see Fig. 2). The second contribution is found by applying the momentum equation to obtain,

$$
\begin{aligned}
\frac{\mathbf{F}_{m}}{\rho b} & =\frac{\left(F_{x}+j F_{y}\right)_{m}}{\rho b} \\
& =-e^{j \Omega} \frac{\partial}{\partial t} \int_{0}^{2 \pi} \int_{R_{1}}^{R_{2}}\left(v_{r}^{\prime \prime}+j v_{\theta}^{\prime \prime}\right) e^{j \theta^{\prime \prime}} r^{\prime \prime} d r^{\prime \prime} d \theta^{\prime \prime} \\
& -\left.e^{j \Omega t}\left[\int_{0}^{2 \pi}\left(v_{r}^{\prime \prime}+j v_{\theta}^{\prime \prime}\right) v_{r}^{\prime \prime} e^{j \theta^{\prime \prime}} r^{\prime \prime} d \theta^{\prime \prime}\right]\right|_{R_{1}} ^{R_{2}} \\
& -e^{j \Omega t} 2 \Omega \int_{0}^{2 \pi} \int_{R_{1}}^{R_{2}}\left(j v_{r}^{\prime \prime}-v_{\theta}^{\prime \prime}\right) e^{j \theta^{\prime \prime}} r^{\prime \prime} d r^{\prime \prime} d \theta^{\prime \prime} \\
& +\omega^{2} \epsilon \pi\left(R_{2}^{2}-R_{1}^{2}\right) e^{j \omega t} .
\end{aligned}
$$

When the pressure distributions of equations (6) and (7), and the velocity profiles described by equations (3)-(5) are applied to equations (20) and (21), the resulting force on the impeller is,

$$
\mathbf{F}^{*}=\mathbf{F}_{p}^{*}+\mathbf{F}_{m}^{*}=\overline{\mathbf{F}}+\epsilon^{*}\left(\mathbf{F}_{c} \cos \omega t+\mathbf{F}_{s} \sin \omega t\right),
$$

where,

$$
\begin{aligned}
& \overline{\mathbf{F}}=\phi^{2}\left[W_{i}^{*} \sec ^{2} \gamma+\mathbf{k} R-2+2 j \tan \gamma\right] \int_{0}^{2 \pi} \bar{\beta}^{2}\left(\theta_{2}\right) e^{j \theta_{2}} d \theta_{2} / 2 \pi \\
& -j \phi\left[W_{i}^{*} \sec ^{2} \gamma \ln (R)+1\right] \int_{0}^{2 \pi} \bar{\beta}\left(\theta_{2}\right) e^{j \theta_{2}} d \theta_{2} / \pi, \\
& \mathbf{F}_{c}=\phi^{2}\left[W_{i}^{*} \sec ^{2} \gamma+\mathbf{k} R-2+2 j \tan \gamma\right] \int_{0}^{2 \pi} \bar{\beta}\left(\theta_{2}\right) \beta_{c}\left(\theta_{2}\right) e^{j \theta_{2}} d \theta_{2} / \pi \\
& -j \phi\left[W_{i}^{*} \sec ^{2} \gamma \ln (R)+1\right] \int_{0}^{2 \pi} \beta_{c}\left(\theta_{2}\right) e^{j \theta_{2}} d \theta_{2} / \pi \\
& +\frac{\omega}{\Omega} \phi\left[W_{i}^{*} \sec ^{2} \gamma \ln (R)+\mathbf{k} / R-1\right] \int_{0}^{2 \pi} \beta_{s}\left(\theta_{2}\right) e^{j \theta_{2}} d \theta_{2} / \pi \\
& +\frac{\omega}{\Omega}\left[\phi R W_{i}^{*} \int_{0}^{2 \pi} \bar{\beta}\left(\theta_{2}\right) \sin \left(\theta_{2}+\tan \gamma \ln (R)\right) e^{j \theta_{2}} d \theta_{2} / \pi\right] \\
& -\frac{\omega}{\mathbf{\Omega}}\left[2 j \phi+W_{i}^{*} /(\mathbf{k} R)\right] \\
& -\frac{\omega^{2}}{\Omega^{2}}\left\{W_{i}^{*}[1-1 /(\mathbf{k} R)] / \tan ^{2} \gamma-1+1 / R^{2}\right\}, \\
& \mathbf{F}_{s}=\phi^{2}\left[W_{i}^{*} \sec ^{2} \gamma+\mathbf{k} R-2+2 j \tan \gamma\right] \int_{0}^{2 \pi} \bar{\beta}\left(\theta_{2}\right) \beta_{s}\left(\theta_{2}\right) e^{j \theta_{2}} d \theta_{2} / \pi \\
& -j \phi\left[W_{i}^{*} \sec ^{2} \gamma \ln (R)+1\right] \int_{0}^{2 \pi} \beta_{s}\left(\theta_{2}\right) e^{j \theta_{2}} d \theta_{2} / \pi \\
& -\frac{\omega}{\Omega} \phi\left[W_{i}^{*} \sec ^{2} \gamma \ln (R)+\mathrm{k} / R-1\right] \int_{0}^{2 \pi} \beta_{c}\left(\theta_{2}\right) e^{j \theta_{2}} d \theta_{2} / \pi \\
& -\frac{\omega}{\Omega}\left[\phi R W_{i}^{*} \int_{0}^{2 \pi} \bar{\beta}\left(\theta_{2}\right) \cos \left(\theta_{2}+\tan \gamma \ln (R)\right) e^{j \theta_{2}} d \theta_{2} / \pi\right] \\
& -\frac{\omega}{\Omega} j\left[2 j \phi+W_{i}^{*} /(\mathbf{k} R)\right] \\
& -\frac{\omega^{2}}{\Omega^{2}} j\left\{W_{i}^{*}[1-1 /(\mathbf{k} R)] / \tan ^{2} \gamma-1+1 / R^{2}\right\},
\end{aligned}
$$

.




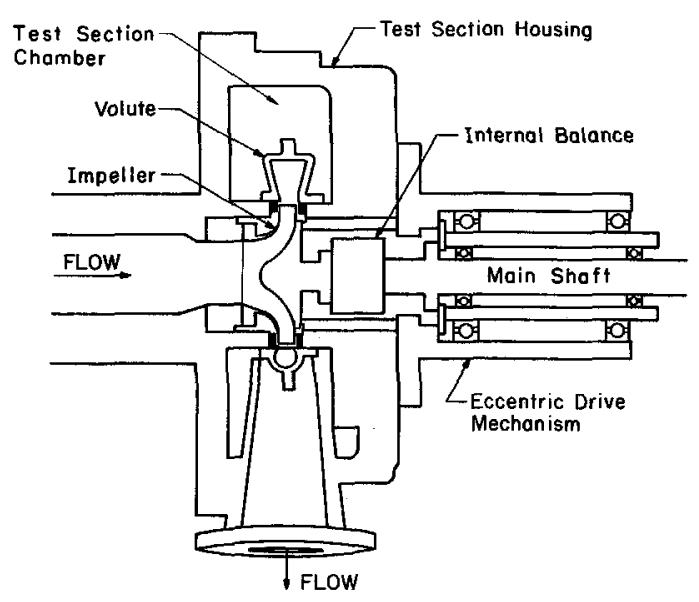

Fig. 4 Schematic of the test section
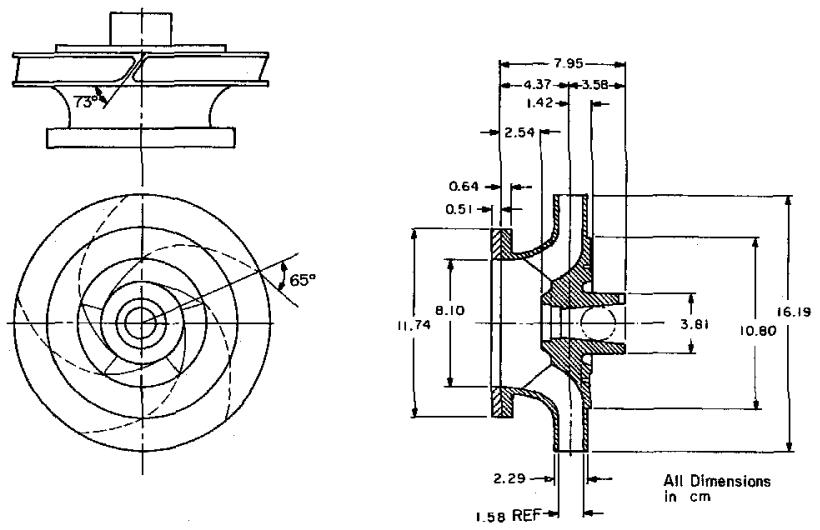

Fig. 5 Dimensions of Impeller $X$


Fig. 6 Dimensions of Volute A

Descriptions of the test impeller and volute are given in Figs. 5 and 6 . The impeller (referred to as Impeller $X$ ) is a five bladed cast bronze impeller with a specific speed of 0.57 and blade angle of $65 \mathrm{deg}$. The $86 \mathrm{deg}$ spiral volute (Volute A) is constructed of fiberglass and designed to be "well matched" with Impeller $X$ at a flow coefficient of 0.092 . The dimensions of the volute cross-sections, shown in Fig. 6, were used in evaluating the integrals of equations $(14 a-e)$.

For this study, the test facility has been modified slightly from the configuration used by Chamieh [1983] and Jery et al. [1984]. The modifications shown in Fig. 7 were considered necessary in order to isolate the interaction between the impeller and the volute from external influences. To separate the flow in the volute from the annular gap region, rings were in-

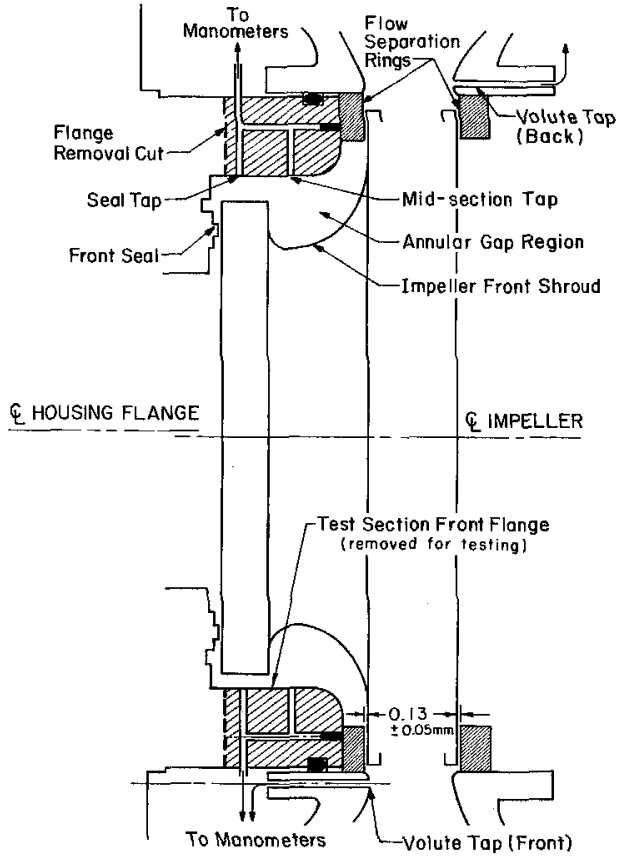

Fig. 7 Modifications of the test facility

stalled $0.13 \mathrm{~mm}$ from the edge of the impeller. In addition, the front flange of the test section was removed so that the front shroud of the impeller was exposed to the "reservoir-like" conditions of the test chamber (see Fig, 4).

The removal of the front flange of the test section was judged to be essential after pressure measurements were made in the annular gap region with the flange in place and the rings removed. The measurements indicated that the fluid trapped in this region was responsible for a hydrodynamic stiffness (see equation (26)) given approximately by,

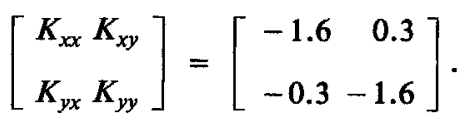

When compared with Chamieh's (1982) direct measurements of the total hydrodynamic stiffness on the impeller (annular gap plus volute) given approximately by,

$$
\left[\begin{array}{l}
K_{x x} K_{x y} \\
K_{y x}
\end{array} K_{y y}\right]=\left[\begin{array}{rr}
-2.0 & 0.9 \\
-0.9 & -2.0
\end{array}\right],
$$

it is seen that the contribution from the annular gap is significant. Impeller shroud forces also had a dominant influence on the hydrodynamic stiffnesses measured by Jery et al. [1984]. With the flange removed, the fluid forces on the front shroud of the impeller were largely eliminated.

\section{Comparisons Between Experimental and Theoretical Results}

The numerical results in this paper can be duplicated using Impeller X properties given in Fig. 5, the Volute A properties in Fig. 6, and the pump performance curves in Fig. 8. A preliminary step in the theoretical calculations is to obtain the impeller flow path angle, $\gamma$ (see Closure Conditions). By setting $\bar{\psi}=\psi_{\text {exp }}$, the flow path angle, $\gamma$, shown in Fig. 9 was obtained. Note that the typical magnitude of $\gamma$ is about $80 \mathrm{deg}$ while the blade angle of Impeller X is $65 \mathrm{deg}$.

Measurements of the static pressure at the discharge from the impeller were made using holes drilled at the inlet to the volute (see Figs. 6 and 7). The circumferential pressure distributions are compared with the theoretical results in Figs. 
10 and 11. The pressure taps are alternately located in the front and back of the volute, resulting in the slight oscillation of the data. The results were obtained for a range of shaft speeds from $\Omega=800$ to $1200 \mathrm{rpm}$, but the nondimensionalized pressures were found to be independent of the speed. Figure 10 shows that the theory gives a good approximation of the pressure distributions over a moderate range of flow coefficients. However, for flow coefficients below this range, the



Fig. 8 Total head coefficient versus the flow coeificient for the Impeller $X$ and Volute $A$ pump. The solid line represents the average value of the experimental data. (Uncertainty in $\psi= \pm 1.5 \times 10^{-3}$, in $\phi= \pm 2$ $\times 10^{-3}$ )

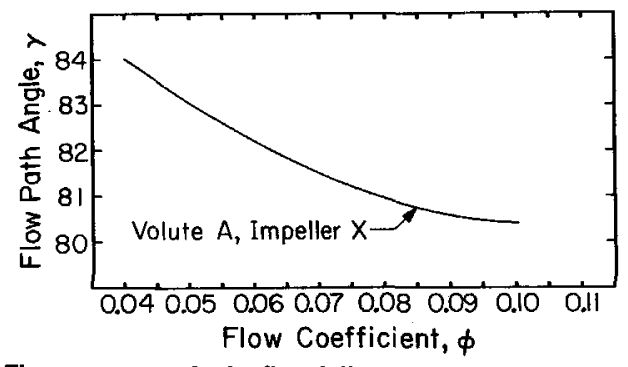

Fig. 9 The average angle the flow follows through the pump (measured with respect to the radial line) as a function of flowrate

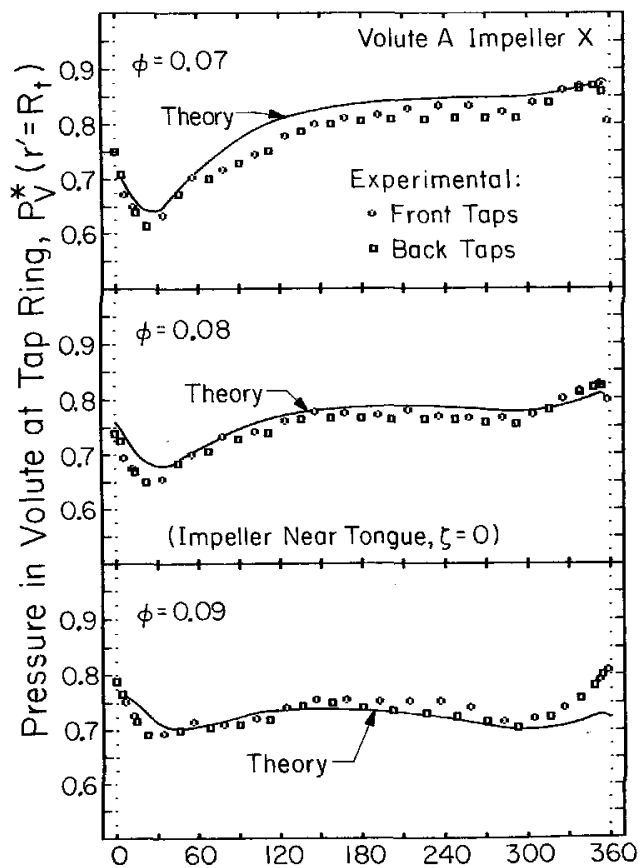

Angle from the Tongue, $\theta^{\prime}$ (degrees)

Fig. 10 Experimental and theoretical pressure distributions at the inlet of the volute for three different flowrates with the impeller placed near the tongue. Measurements were taken with $\Omega=1000 \mathrm{rpm}$. (Uncertainty in $P_{v}^{*}= \pm 3 \times 10^{-4}$, in $\phi= \pm 2 \times 10^{-3}$, in $\theta= \pm 0.25 \mathrm{deg}$, in $\zeta=$ \pm 0.5 deg) pressure predictions begin to falter as shown in Fig. 11. The model's assumption of log spiral stream paths through the impeller probably fails at lower flowrates. For higher flow coefficients, it was concluded that the deviation was caused by the inadequacy of a one dimensional treatment of the flow near the tongue of the volute. At the higher flowrates, it has been suggested (Lazarkiewicz and Troskolanski, 1965) that there is a reversal of the direction of flow in the region just inside the tongue. The effect on the pressure distribution of displacing the impeller is also demonstrated in Fig. 11. The model appears to follow the changes that occur, even when the absolute pressure predictions are rather poor.

A comparison between the experimental and theoretical steady forces on the impeller is given in Fig. 12. One set of experimental results was obtained by placing the impeller in four equally spaced orbit positions and then averaging the internal balance force measurements. The second set of experimental results (for $\phi=0.06$ and 0.10 ) was obtained by integrating the discharge pressure measurements. The theoretical model tends to overpredict the steady or average radial forces somewhat, but it does give reasonable results considering the crudeness of the model. Colding-Jorgensen's (1980) steady force calculations for a $67.5 \mathrm{deg}$ blade angle impeller in an $86 \mathrm{deg}$ spiral volute are also shown in Fig. 12. The present model appears to give a more accurate assessment of the measured steady forces. The agreement between the two sets of experimental data indicates that the primary cause of the radial forces is the asymmetric pressure distribution at the discharge of the impeller. Moreover, the theoretical model predicted that the discharge pressure was responsible for 99 percent of the total force on the impeller while the net momentum flux contribution was essentially negligible. It might also be of interest to note that over the entire range of flowrates for which theoretical results are presented, the predicted perturbation in the impeller discharge flow never exceeded 6 percent of the mean flow.

Figure 13 presents the components of the generalized hydrodynamic force matrix, $[A]$, that results when the impeller whirls in an eccentric orbit at the pump design flowrate

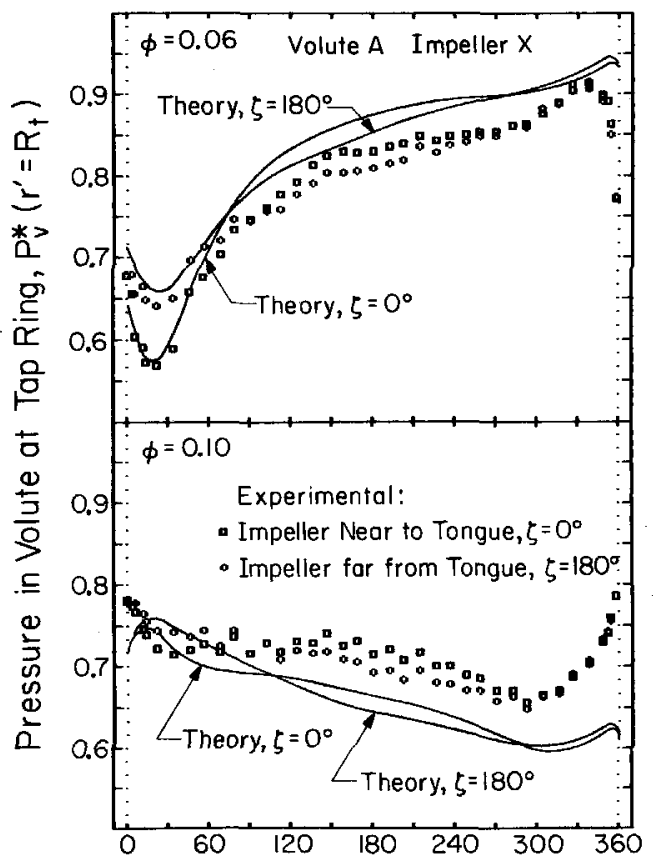

Angle from the Tongue, $\theta^{\prime}$ (degrees)

Fig. 11 Experimental and theoretical pressure distributions at the inlet of the volute for two different flowrates with the impeller placed near to and far from the volute tongue. (Uncertainty in $P_{y}^{*}= \pm 3 \times 10^{-4}$, in $\phi=$ $\pm 2 \times 10^{-3}$, in $\theta= \pm 0.25 \mathrm{deg}$, in $\zeta= \pm 0.5 \mathrm{deg}$ ) 


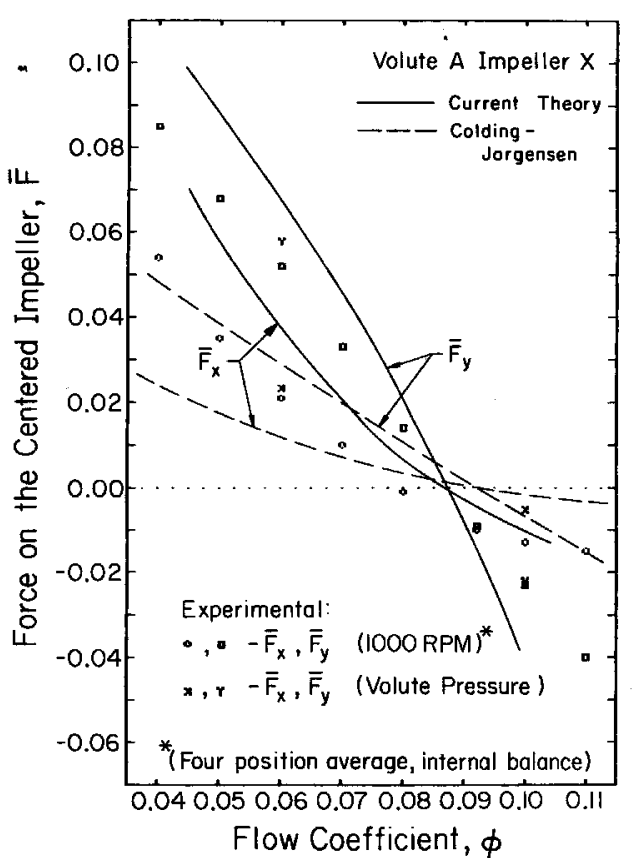

Flg. 12 Steady hydrodynamic forces acting upon Impeller $X$ as function of flowrate. Experimental forces were obtained by both internal balance and discharge pressure measurements. Colding-Jorgensen's (1980) results are for an 86 deg spiral volute with a 67.5 deg blade angle impeller. (Uncertainty in $F= \pm 3 \times 10^{-3}$, in $\phi= \pm 2 \times 10^{-3}$, in rpm $=$ $\left.\pm 10^{-3} \mathrm{rpm}\right)$

( $\phi=0.092)$. Cross-coupled terms (i.e., $A_{x y}, A_{y x}$ ) in the experimental data suggest that forces act in the direction of the whirl orbit up to $\omega / \Omega=0.10$. This destabilizing influence is predicted by the theoretical model to occur up to $\omega / \Omega=0.14$. Due to the coupled nature of equations $(7 b)$ and $(7 c)$, it was not possible to calculate $[A]$ beyond the range of whirl ratios shown in Fig. 13. This problem is believed to result from current limitations of the iterative technique used in obtaining a numerical solution.

As was mentioned in the introduction, it is a standard practice to express the matrix elements of $[A]$ in powers of $\omega$. By examining the $A_{y x}$ term in Fig. 13, it is apparent that a quadratic in $\omega$ will not adequately describe the features of the matrix element. However, a cubic in $\omega$ can approximate all of the $[A]$ matrix element variations with $\omega$. The coefficients of such an expansion can be written as,

$\left[\begin{array}{cc}A_{x x} & A_{x y} \\ A_{y x} & A_{y y}\end{array}\right]=$

$\left[\begin{array}{ll}-K_{x x}-\omega C_{x y}+\omega^{2} M_{x x}+\omega^{3} J_{x y} & -K_{x y}+\omega C_{x x}+\omega^{2} M_{x y}-\omega^{3} J_{x x} \\ -K_{y x}-\omega C_{y y}+\omega^{2} M_{y x}+\omega^{3} J_{y y} & -K_{y y}+\omega C_{y x}+\omega^{2} M_{y y}-\omega^{3} J_{y x}\end{array}\right]$

or alternatively as,

$\begin{aligned} {[A(\omega / \Omega)]\left\{\begin{array}{l}x \\ y\end{array}\right\}=} & -[K]\left\{\begin{array}{l}x \\ y\end{array}\right\}-[C]\left\{\begin{array}{l}\dot{x} \\ \dot{y}\end{array}\right\} \\ & -[M]\left\{\begin{array}{l}\ddot{x} \\ \ddot{y}\end{array}\right\}-\left[J\left\{\begin{array}{l}\ddot{x} \\ \dddot{y}\end{array}\right\},\right.\end{aligned}$

where,

$$
x=\epsilon \cos \omega t \text { and } y=\epsilon \sin \omega t .
$$

The $[K],[C]$, and $[M]$ matrices correspond to the stiffness, damping, and inertial components that are commonly

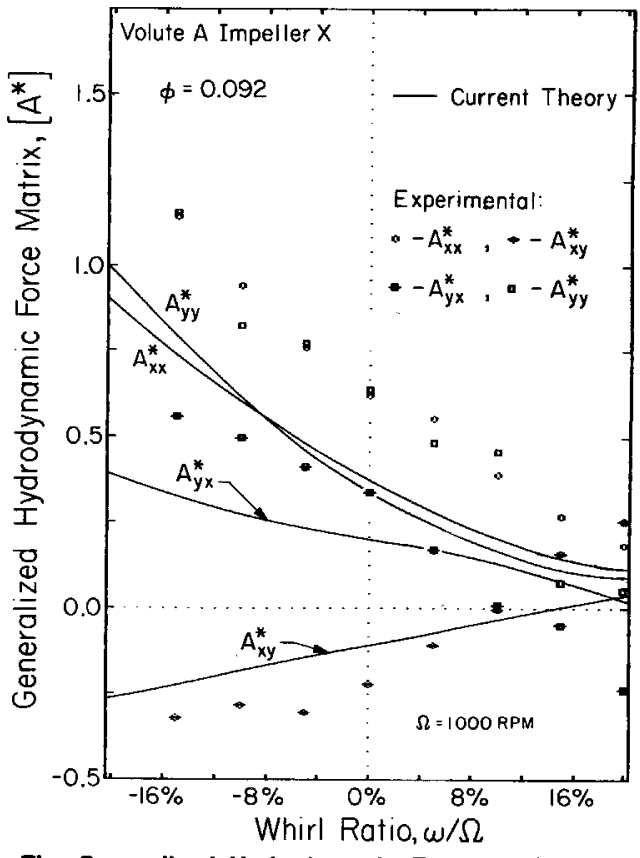

Fig. 13 The Generalized Hydrodynamic Force coefficients as functions of whirl speed at the volute design flow coefficient ( $\phi=0.092$ ) The experimental results were obtained from the direct force measurements of the internal balance. The radius of the whirl orbit for Impeller X was $\epsilon=2.51 \mathrm{~mm}$. (Uncertainty in $A^{*}= \pm 3 \times 10^{-3}$, in $\omega / \Omega=$ $10^{-2}$, in $\phi= \pm 2 \times 10^{-3}$ )

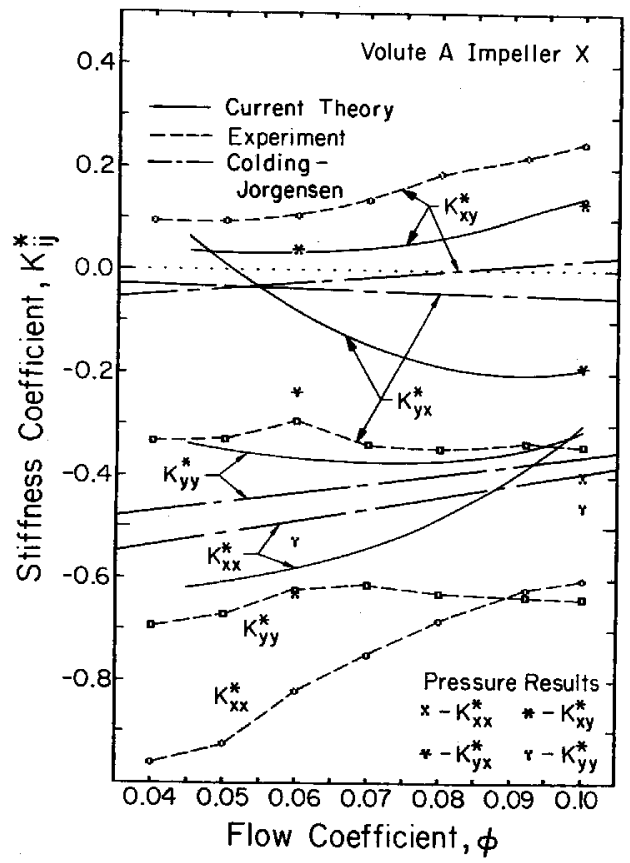

Fig. 14 Hydrodynamic stiffnesses as functions of the flowrate. Experimental results are from internal balance and pressure measurements. Colding-Jorgensen's (1980) results are for an 86 deg spiral volute with a 67.5 deg blade angle impeller. (Uncertainty in $K^{*}=$ $\pm 3 \times 10^{-3}$, in $\phi= \pm 2 \times 10^{-3}$ )

employed in rotordynamics. Since the $[J]$ matrix is related to the third order time derivative of the impeller displacement (which is conventionally known as the jerk), it will be referred to as the "jerk" matrix.

The resulting $[K]$ matrix elements of the cubic expansion are given in Fig. 14, and the $[C],[M]$, and $[J]$ matrix elements are presented in Fig. 15. Included in Fig. 14 are the stiffnesses that were calculated using the force measurements (from the internal balance and the pressure distributions) taken at four impeller positions. Also shown in Fig. 14 are the stiffnesses 




Fig. 15 Damping, mass, and jerk force coefficients of the flowrate as predicted by the current theoretical model

predicted by Colding-Jorgensen (1980) for an 86 deg spiral volute. With the exception of the $K_{y x}$ term, the current model does a fair job of describing the variation of stiffness with flowrate. The magnitudes, however, tend to be underpredicted by the theory. Over most of the range of flow coefficients, the stiffness is such that it would encourage the whirling motion of the impeller. The same is also true of the damping when the flowrate drops below $\phi=0.07$ as shown in Fig. 15. The magnitude of the damping components computed by ColdingJorgensen (1980) were less than 10 percent of those predicted by the present model. In general, the inertial force would discourage an orbital motion of the impeller, but it will tend to drive the impeller in the direction of the displacement. The jerk force attains significant values only at the lower flow coefficients. However it must be emphasized that the damping, mass and jerk components have not been experimentally validated.

\section{Closing Comments}

A theoretical model has been developed to describe the flow in the impeller and the volute, along with the interactions that occur between them. This investigation was undertaken to provide a better understanding of the destabilizing hydrodynamic forces that have been observed by Chamieh et al. (1982) and Jery et al. (1984) on a whirling centrifugal pump impeller. To implement the model requires only a knowledge of the dimensions of the volute and impeller, and the total head rise across the entire pump. Comparisons between the predicted and experimental results are encouraging.
Experimentation with different volute geometries and over a wider range of operating conditions (flow coefficient and whirl ratio) would provide a more crucial test of the theoretical model. It might also be useful to incorporate the effects of inducers and diffuser vanes into the theoretical model. These devices are now commonly employed on many high performance centrifugal pumps.

Previous experimental results (Chamieh et al., 1982, and Jery et al., 1984) have tended to over-estimate the contribution of the volute/impeller interaction to the total stiffness force acting on the impeller. The over-estimation came about because of an asymmetric pressure distribution in the fluid trapped on the front shroud of the impeller. Since real pumps do have fluid in this region, it will be important in the future to perform a detailed study of this area. Some work is beginning to appear in this important area (Childs, 1986).

\section{Acknowledgments}

This research was funded by NASA George Marshal Space Flight Center, Huntsville, Alabama under contract NAS 8-33108. Both of the authors would like to express their gratitude to A. J. Acosta and T. K. Caughey for their helpful advice, and R. Franz and N. Ardnt for their assistance with the experiments. One of the authors (D. R. Adkins) would also like to thank the Shell Companies Foundation for a fellowship that supported his graduate studies and Susan Berkley and Jan Patterson for their invaluable help in preparing this paper.

\section{References}

Adkins, D. R., 1985, “Analyses of Hydrodynamic Forces on Centrifugal Pump Impellers," Ph.D. thesis, Division of Engineering and Applied Sciences, California Institute of Technology.

Agostinelli, A., Nobles, D., and Mockridge, C. R., 1960, "An Experimental Investigation of Radial Thrust in Centrifugal Pumps," ASME Journal of Engineering for Power, Vol. 82, pp. 120-126.

Brennen, C. E., Acosta, A. J., and Caughey, T. K., 1980, "A Test Program to Measure Fluid Mechanical Whirl-Excitation Forces in Centrifugal Pumps," First Workshop on Rotordynamic Instability Problems in High Performance Turbomachinery, Texas A\&M University, NASA Conf. Pub. 2133, pp. 229-235.

Chamieh, D. S., 1983, "Forces On A Whirling Centrifugal Pump-Impeller," Ph.D. thesis, Division of Engineering and Applied Sciences, California Institute of Technology.

Chamieh, D. S., Acosta, A. J., Brennen, C. E., Caughey, T. K., and Franz, R., 1982, "Experimental Measurements of Hydrodynamic Stiffness Matrices for a Centrifugal Pump Impeller," 2nd Workshop on Rotordynamic Instability Problems in High Performance Turbomachinery, Texas A\&M University, NASA Conf. Pub. 2250, pp. 382-398.

Childs, D. W., 1986, "Force and Moment Rotordynamic Coefficients for Pump-Impeller Shroud Surfaces," Rotordynamic Instability Problems in High Performance Turbomachinery, Texas A\&M University, NASA Conf. Pub. 2443, pp. 503-529.

Colding-Jorgensen, J., 1980, "The Effect of Fluid Forces on Rotor Stability of Centrifugal Compressors and Pumps," First Workshop on Rotordynamic Instability Problems in High Performance Turbomachinery, Texas A\&M University, NASA Conf. Pub. 2133, pp. 249-266.

Csanady, G. T., 1962, "Radial Forces in a Pump Caused by Volute Casing," ASME Journal of Engineering for Power, Vol. 84, pp. 337-340.

Ehrich, F., and Childs, D., 1984, "Self-Excited Vibration in High Performance Turbomachinery," Mechanical Engineering, Vol. 106, No. 5, pp. 66-79.

Iversen, H. W., Rolling, R. E., and Carlson, J. J., 1960, "Volute Pressure Distribution, Radial Force on the Impeller and Volute Mixing Losses of a Radial Flow Centrifugal Pump," ASME Journal of Engineering for Power, Vol, 82, pp. 136-144.

Jery, B., Acosta, A. J., Brennen, C. E., and Caughey, T. K., 1984, "Hydrodynamic Impeller Stiffness, Damping and Inertia in the Rotordynamics of Centrifugal Flow Pumps," Rotordynamic Instability Problems in High Performance Turbomachinery, Texas A\&M University, NASA Conf. Pub. 2338, pp. 137-160.

Lazarkiewicz, S., and Troskolanski, A. T., 1965, Impeller Pumps, Pergamon Press, Translated by D. K. Rutter (Polish Ed. Title: Pompy Wirowe).

Shoji, H., and Ohashi, H., 1980, "Fluid Forces on Rotating Centrifugal Impeller with Whirling Motion," NASA CP 2133, pp. 317-328.

Shoji, H., and Ohashi, H., 1987, "Lateral Forces on Whirling Centrifugal Impeller (1st Report: Theory), ASME Journal of Fluids EngineERING, Vol. 109, No. 2, pp. 94-99. 